\title{
Mode-Selected Electron-Phonon Coupling in Superconducting Pb Nanofilms Determined from He Atom Scattering
}

\author{
I. Yu. Sklyadneva, ${ }^{1,2}$ G. Benedek, ${ }^{1,3, *}$ E. V. Chulkov, ${ }^{1,4}$ P. M. Echenique, ${ }^{1,4}$ R. Heid, ${ }^{5}$ K.-P. Bohnen, ${ }^{5}$ and J. P. Toennies ${ }^{6}$ \\ ${ }^{1}$ Donostia International Physics Centre (DIPC), Paseo Manuel de Lardizàbal 4, 20018 Donostia/San Sebastian, Spain \\ ${ }^{2}$ Tomsk State University, 634050 Tomsk, Russian Federation \\ ${ }^{3}$ Dipartimento di Scienza dei Materiali, Università di Milano-Bicocca, Via Cozzi 53, 20125 Milano, Italy \\ ${ }^{4}$ Departamento de Física de Materiales and CFM (CSIC-UPV/EHU), Universidad del País Vasco, \\ 20018 San Sebastián/Donostia, Spain \\ ${ }^{5}$ Karlsruher Institut für Technologie, Institut für Festkörperphysik, P.O. Box 3640, D-76021 Karlsruhe, Germany \\ ${ }^{6}$ Max Planck Institut für Dynamik und Selbstorganisation, Bunsenstrasse 10, 37073 Göttingen, Germany
}

(Received 7 June 2011; published 25 August 2011)

The electron-phonon coupling (EPC) strength for each phonon mode in superconducting $\mathrm{Pb}$ films is measured by inelastic helium atom scattering (IHAS). This surprising ability of IHAS relies on two facts: (a) In ultrathin metal films, the EPC range exceeds the film thickness, thus enabling IHAS to detect most film phonons, even $1 \mathrm{~nm}$ below the surface; (b) IHAS scattering amplitudes from single phonons are shown, by first-principle arguments, to be proportional to the respective EPC strengths. Thus IHAS is the first experiment providing mode-selected EPC strengths (mode-lambda spectroscopy).

DOI: 10.1103/PhysRevLett.107.095502

Many of the unusual physical properties of lead stem from its large electron-phonon coupling (EPC) constant $\lambda=1.12-1.68$. Besides explaining the second-highest critical temperature of all elemental superconductors $\left(T_{c \text { bulk }}=7.23 \mathrm{~K}\right)$, the strong EPC of lead manifests itself in the especially large and numerous Kohn anomalies affecting the bulk phonon dispersion curves. The large EPC also accounts for the occurrence of superconductivity in supported lead films as thin as only a few monolayers [1-5]. The recent discovery of superconductivity in a single lead monolayer on silicon [6] raises intriguing questions about the EPC in quasi-two-dimensional systems. Thin lead films also exhibit important quantum-size oscillations in the layer-by-layer growth [7], first observed by He-atom scattering and attributed to interference with the quantum-well states. The latter modulate the electron density of states at the Fermi level and the EPC, thereby also causing oscillations with thickness in the superconducting critical temperature and the upper critical field [5], interlayer distances [8], island height distributions [9], and zone-center phonon frequencies [10,11], as well as in electronic transport [12], photoemission properties [13], and work functions [14]. Thus lead films have become an important model system for exploring superconductivity and electronic properties of metals in general on the nanoscale.

The present study was initially undertaken to understand why recent inelastic helium atom scattering (IHAS) phonon measurements of thin few monolayer (ML) crystalline films of lead on $\mathrm{Cu}(111)$ [11] exhibited a much larger number of phonon dispersion curves than found for ordinary metal and thin-film surfaces [15]. The multitude of dispersion curves, illustrated in Figs. 1(a) and 1(b) for
PACS numbers: 63.20.kd, 63.22.Dc, 68.49.Bc, 74.78.Fk

the case of 5 and 7 ML lead films, respectively, grown on a $\mathrm{Cu}(111)$ substrate, were obtained with the Göttingen apparatus HUGO I [11] at a surface temperature of $140 \mathrm{~K}$ and an incident He-atom beam energy of $E_{i}=23 \mathrm{meV}$. The dispersion curves in the $\langle 11 \overline{2}\rangle(\bar{\Gamma}-\bar{M})$ surface direction for 5 and $7 \mathrm{ML}$ were extracted from time-of-flight spectra such as those shown in Figs. 1(c) and 1(d), respectively. Here it is shown by first-principle arguments that IHAS scattering amplitudes from single phonons are proportional to the respective EPC strengths and that the extraordinary ability of IHAS of detecting a large number of phonon branches, including those deeply localized several layers beneath the surface, is due to the fact that in ultrathin superconducting films the EPC, besides being large, has a range exceeding the film thickness. This enables IHAS to detect all the film phonons which substantially contribute to superconductivity and to measure their EPC strength (mode-lambda spectroscopy). Besides for understanding superconductivity in ultrathin films, mode-selected EPC strengths are of basic relevance for inelastic photoemission [16], phonon-induced surface reactions [17], scanning tunnel spectroscopy [18], and other thin-film properties $[14,19]$.

Density functional perturbation theory calculations of the dispersion curves were carried out which include also the atomic displacements of all the atoms in the film for each eigenmode. In the same calculation it was also possible to obtain the associated electron-density oscillations (EDOs) throughout the entire film. To keep the computational effort within a reasonable size, the substrate was assumed to be rigid and simulated by a single additional monolayer of $\mathrm{Pb}(111)$ with a virtually infinite nuclear mass. The distance between the bottom layer of the $\mathrm{Pb}$ 

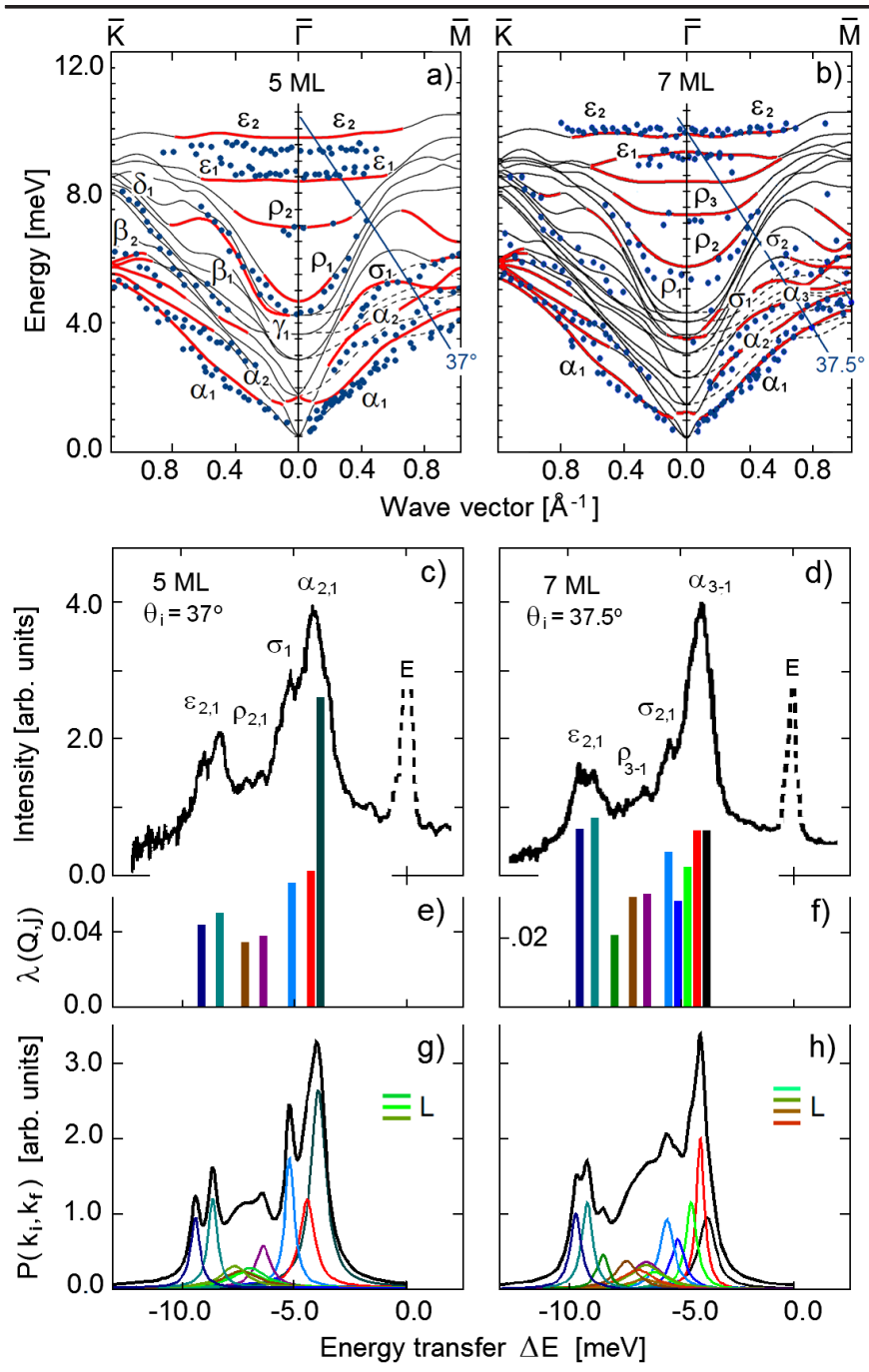

FIG. 1 (color online). The experimental phonon dispersion curves of 5 (a) and $7 \mathrm{ML}$ (b) $\mathrm{Pb}(111)$ films $(T=140 \mathrm{~K})$ on $\mathrm{Cu}(111)$, measured by IHAS at an incident beam energy of $23 \mathrm{meV}$ along two symmetry directions $(\mathbf{)})$, are compared with the density functional perturbation theory calculations. The phonon branches are labeled $\left(\alpha_{i}, \beta_{i}, \ldots ; i=1,2, \ldots\right)$ following the convention of Ref. [11]. Heavy red lines represent the active modes with prevalent SV polarization; broken lines in the $\bar{\Gamma} \bar{M}$ direction are shear-horizontal modes. The oblique lines are typical scan curves for incident angles of $37^{\circ}(5 \mathrm{ML})$ and $37.5^{\circ}$ ( $7 \mathrm{ML}$ ). They serve to assign the most prominent features of the energy loss spectra to the energy and parallel momentum of the phonons [11]. Panels (c) and (d) show the respective energy transfer spectra from time-of-flight measurements at the same angles ( $\Delta E<0$ for phonon creation processes). The diffuse elastic scattering peak $E$ is due to a small concentration of surface defects found on even the most perfectly structured surfaces. The calculated electron-phonon coupling constants $\lambda_{\mathbf{Q} \nu}$ for the $\mathrm{SV}$ modes intersected by the scan curves in (a),(b), indicated by colored bars in (e),(f), and the corresponding inelastic spectra $[(\mathrm{g}),(\mathrm{h})$, heavy line] including both the SV- and the weaker L-mode contributions (Lorentzian components), are in good agreement with the energy transfer spectra (c),(d). The colors for SV modes are the same as in Figs. 3(a) and 3(b). film and the substrate layer was reduced by $5 \%$ in order to reproduce the force constant stiffening at the $\mathrm{Cu}-\mathrm{Pb}$ interface. These calculations were performed by using the mixed-basis pseudopotential method [20] with the local density approximation for the exchange-correlation functional. Structural optimization was carried out to account for the variation of the interlayer distances in the film. The lateral lattice parameter inside the $\mathrm{Pb}(111)$ layers was fixed at the theoretical bulk value $a=9.20$ a.u., which is $1.6 \%$ smaller than the experimental value of 9.35 a.u. The calculated relaxation shows a contraction of the outermost interlayer spacing relative to the bulk distance of $-4.30 \%$ for $5 \mathrm{ML}$ and $-4.25 \%$ for $7 \mathrm{ML}$. Interpolation of a $(12 \times 12)$ $\mathbf{Q}$ point mesh for the hexagonal surface Brillouin zone provided the phonon dispersion curves and polarization vectors [21].

The dispersion curves calculated for 5 and $7 \mathrm{ML}$ are compared with the experimental data in Figs. 1(a) and 1(b). Thick red lines correspond to modes with prevalent shearvertical (SV) polarization, broken lines to modes with a shear-horizontal polarization, which are symmetry forbidden to IHAS along the $\langle 112\rangle$ azimuth. The comparison indicates that nearly all the SV modes of the film are excited and not only the surface modes as in all previous experiments. As seen in Fig. 2, the topmost mode $\varepsilon_{2}$ has a much larger atom displacement in the first two surface layers than the $\varepsilon_{1}$ mode. According to the standard surface atom impulsive excitation mechanism, $\varepsilon_{2}$ should have a much larger probability for excitation. The comparable intensities of the $\varepsilon_{1,2}$ doublet reveal the unexpected ability of IHAS to detect phonons localized several layers beneath the surface.

The puzzle is solved by the calculation of the EDOs for the two modes (Fig. 2): It appears that their amplitudes at the classical He-atom turning point, $\zeta_{\mathrm{ti}}=3.5 \AA$ above the topmost atomic layer, are about equal despite the large difference in the displacement patterns. This strongly suggests that the interaction of the $\mathrm{He}$ atoms with the phonons of the film must be mediated by the electron-phonon interaction responsible for the phonon-induced EDOs.

Theory shows that the IHAS intensities are directly proportional to the respective mode-selected EPC strengths. A detailed derivation is found in the Supplemental Materials [22]. The He-surface scattering potential is assumed proportional to the surface charge density $n(\mathbf{r})$ at the classical turning point: $V(\mathbf{r})=A n(\mathbf{r})$ [23]. Correspondingly, phonon-induced modulation is given by $\delta V(\mathbf{r}, t)=$ $A \delta n(\mathbf{r}, t)$. The inelastic scattering probability $P\left(\mathbf{k}_{i}, \mathbf{k}_{f}\right)$ for a He atom in an initial state $\langle i|$ of wave vector $\mathbf{k}_{i} \equiv$ $\left(\mathbf{K}_{i}, k_{i \zeta}\right)$ and energy $E_{i}$ to be excited to a final state $|f\rangle$ of wave vector $\mathbf{k}_{f} \equiv\left(\mathbf{K}_{f}, k_{f \zeta}\right)$ and energy $E_{f}=E_{i}-\Delta E$ via the creation of one phonon of parallel wave vector $\mathbf{Q}=$ $\mathbf{K}_{f}-\mathbf{K}_{i}$, and energy $\varepsilon_{\mathbf{Q} \nu}$, is accurately described, for a weakly corrugated metal surface, by the distorted wave Born approximation [15]: 


$$
\begin{aligned}
P\left(\mathbf{k}_{i}, \mathbf{k}_{f}\right) \propto & \left|\frac{k_{f}}{k_{i \zeta}} n_{\mathrm{BE}}(-\Delta E)\right| \\
& \times \sum_{\mathbf{Q} v} \sum_{\mathbf{K} n}\left|\delta V_{\mathrm{fi}}(\mathbf{K} n, \mathbf{Q} \nu)\right|^{2} \delta\left(\Delta E-\varepsilon_{\mathbf{Q} \nu}\right),
\end{aligned}
$$

where $n_{\mathrm{BE}}(\Delta E)$ is the Bose-Einstein occupation number. $\delta V_{\mathrm{fi}}(\mathbf{K} n, \mathbf{Q} \nu)$ is the matrix element between initial and final He-atom states of the potential modulation due to all the virtual electronic transitions induced by a phonon of wave vector $\mathbf{Q}$ and branch index $\nu$ from a given quantumwell state $\psi_{\mathbf{K} n}(\mathbf{r})$ of parallel wave vector $\mathbf{K}$ and band index $n$ to any other quantum-well state $\psi_{\mathbf{K}+\mathbf{Q} n^{\prime}}(\mathbf{r})$ across the Fermi level. Following Grimvall [24], $\delta V_{\mathrm{fi}}(\mathbf{K} n, \mathbf{Q} \nu)$ can be expressed through the EPC matrix $g_{n n^{\prime}}(\mathbf{K}, \mathbf{K}+\mathbf{Q} ; \mathbf{Q} \nu)$ as

$$
\begin{aligned}
\delta V_{\mathrm{fi}}(\mathbf{K} n, \mathbf{Q} \nu)= & A\left\langle f\left|\delta n_{\mathbf{K} n, \mathbf{Q} \nu}(\mathbf{r})\right| i\right\rangle \\
= & -\frac{A}{\varepsilon_{\mathbf{Q} \nu}} \sum_{n^{\prime}} g_{n n^{\prime}}(\mathbf{K}, \mathbf{K}+\mathbf{Q} ; \nu) \\
& \times\left\langle f\left|\psi_{\mathbf{K} n}^{+}(\mathbf{r}) \psi_{\mathbf{K}+\mathbf{Q} n^{\prime}}(\mathbf{r})\right| i\right\rangle,
\end{aligned}
$$

where $\delta n_{\mathbf{K} n, \mathbf{Q} \nu}(\mathbf{r})$ is the corresponding electron-density modulation component and $\varepsilon_{\mathbf{Q} \nu}$ the phonon energy. As shown in [22], the matrix element on the right-hand side of Eq. (2) is a function, designated as $I\left(\varepsilon_{\mathrm{Q} \nu}\right)$, which depends approximately only on the phonon energy. Clearly, the products of the two electronic wave functions, weighed by the respective EPC matrix elements and multiplied by $-A / \varepsilon_{\mathbf{Q} v}$, act as components of the inelastic scattering potential. After inserting Eq. (2) into Eq. (1), the result is simplified by introducing the EPC constants for each individual phonon (the mode lambda) [25]:

$\lambda_{\mathbf{Q} \nu} \equiv\left[2 \hbar / \varepsilon_{\mathbf{Q} \nu}^{3} N\left(\varepsilon_{F}\right)\right] \sum_{\mathbf{K} n}\left|\sum_{n^{\prime}} g_{n n^{\prime}}(\mathbf{K}, \mathbf{K}+\mathbf{Q} ; \nu)\right|^{2}$,

where $N\left(E_{F}\right)$ is the density of states at the Fermi energy $E_{F}$. In Eq. (1), the sum of $\left|\delta V_{\mathrm{fi}}(\mathbf{K} n, \mathbf{Q} \nu)\right|^{2}$ over all quantumwell states $\mathbf{K} n$ at the Fermi level yields the inelastic scattering probability (see Fig. S1 of the Supplemental Materials [22]) giving

$$
P\left(\mathbf{k}_{i}, \mathbf{k}_{f}\right) \propto \sum_{\mathbf{Q} \nu} f(\Delta E) \lambda_{\mathbf{Q} \nu} \delta\left(\Delta E-\varepsilon_{\mathbf{Q} \nu}\right),
$$

with $f(\varepsilon)=\left|\varepsilon n_{\mathrm{BE}}(-\varepsilon) k_{f} / k_{i \zeta}\right| I^{2}(\varepsilon)$. Since the kinematic factor $f(\Delta E)$ is found to depend rather weakly on the energy transfer $\Delta E$, to a good approximation the IHAS peak intensities for individual phonons are proportional to the respective EPC strengths $\lambda_{\mathbf{Q} \nu}$.

To test this result the coupling constants $\lambda_{\mathbf{Q} \nu}$ were calculated $a b$ initio as in Ref. [25] for 5 and 7 ML films as functions of $\mathbf{Q}$ in the $\bar{\Gamma}-\bar{M}=\langle 11 \overline{2}\rangle$ direction for the phonons with a prevalent SV character. The predicted EPC constants $\lambda_{\mathbf{Q} \nu}$ for all 7 (5 ML) and 10 (7 ML) experimentally resolved modes are plotted in Figs. 3(a) and 3(b) as a function of the wave vector. In Figs. 1(e) and 1(f), the $\lambda_{\mathbf{Q} \nu}$ are plotted for the specific phonons as bar diagrams for a
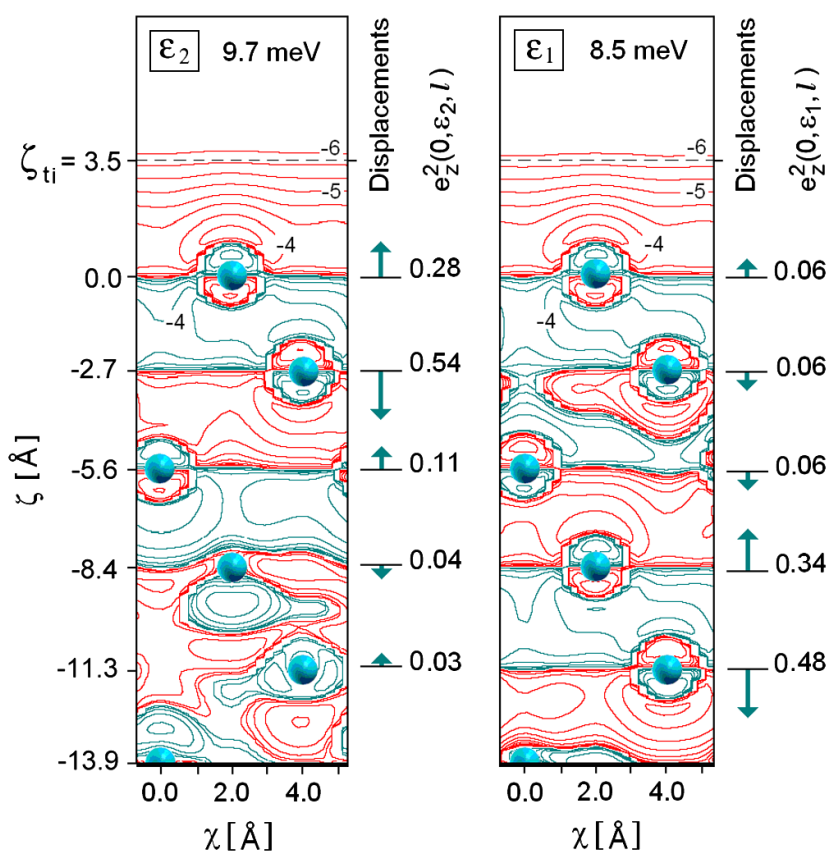

FIG. 2 (color online). The atomic core displacements of the $\varepsilon_{1}$ and $\varepsilon_{2}$ phonon modes at zero wave vector of a 5 monolayer lead film on a rigid substrate induce the EDOs shown in the figure. The contour lines are for equal EDO amplitudes $\pm 10^{-n}$ atomic units ( + for red, - for turquoise lines) and are labeled by the exponent $n$. The core displacement amplitudes are shown by vertical arrows to the right of each of the contour plots, together with the corresponding square eigenvectors. The heavy line at $-13.9 \AA$ indicates the location of the rigid substrate. Although the $\varepsilon_{2}$ mode is mostly localized near the surface and $\varepsilon_{1}$ near the substrate layer, both modes have almost equal EDOs at a distance from the surface corresponding to the classical He-atom turning point $\zeta_{\mathrm{ti}}=3.5 \AA$ above the topmost atomic layer, thus explaining their comparable IHAS signals. In the conventional atom-atom collision model, the IHAS intensity for the $\varepsilon_{2}$ mode would be about 5 times larger than for the $\varepsilon_{1}$ mode.

direct comparison with the respective energy loss spectra of Figs. 1(c) and 1(d). The very satisfactory agreement provides convincing support for the theory and the approximations made. Similar results are obtained for other incident angles. The correspondence between experiment and theory is even more convincing if the contributions of all sagittal [SV and quasilongitudinal (L)] modes are included in the simulations [Figs. 1(g) and 1(h)] with the calculated factor $f(\Delta E)$ and the experimental width corrected by the Jacobian factor due to the intersection angle between scan and dispersion curves. As explained in the Supplemental Materials [22], SV modes couple to interband $\left(n \neq n^{\prime}\right)$ transitions, whereas L modes preferentially couple to intraband $\left(n=n^{\prime}\right)$ transitions. As a consequence, the role of $\mathrm{L}$ modes decreases for an increasing number of layers.

These results lead to the important conclusion that IHAS can be used for a direct measurement of mode-selected $e-p$ coupling constants in conducting films, notably in 


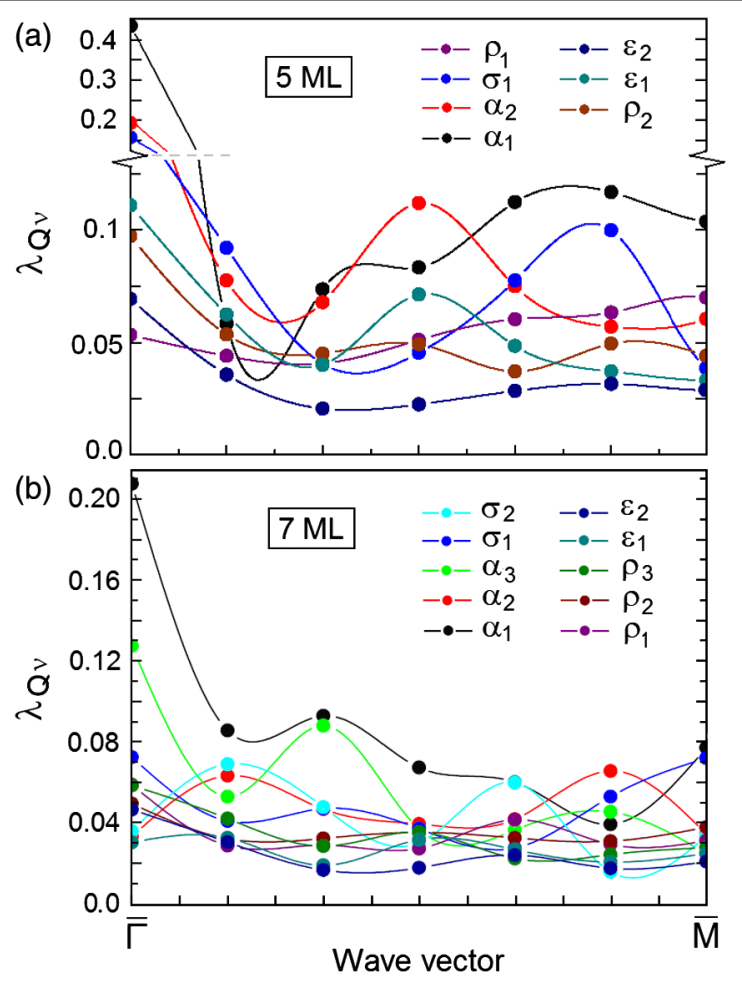

FIG. 3 (color online). Calculated values of the mode-selected electron-phonon coupling constants $\lambda_{\mathbf{Q} \nu}$ for modes of quasi-SV polarization, as functions of the two-dimensional wave vector in the $\bar{\Gamma}-\bar{M}$ symmetry direction, for $5 \mathrm{ML}$ (a) and $7 \mathrm{ML}$ (b) $\mathrm{Pb} / \mathrm{Cu}(111)$ films. The mode labels are the same as those in Figs. 1(a) and 1(b) and the colors as in Figs. 1(e) and 1(f).

superconductors and other low-dimensional systems with strong manifestations of the electron-phonon interaction. A mode-specific spectroscopy of electron-phonon coupling constants can now be envisaged with much higher resolution, recently available in ${ }^{3} \mathrm{He}$ inelastic surface scattering with the spin-echo technique (energy resolution $20 \mathrm{neV}$ versus $0.1 \mathrm{meV}$ with time of flight) [26]. An example of the type of valuable information which might become accessible is the identification of the phonons which are most efficient in the formation of Cooper pairs in BCS thin-film superconductors. These results also indicate a way to exploring phonon waves propagating at the interface of two elastic media, which in seismology are known as Stonely waves. Finally, we note that phonon-induced EDOs can be expected to play an important role in understanding phonon-induced reactive scattering, especially of open shell atoms and molecules at metal surfaces [17].

One of us (G. B.) acknowledges the Ikerbasque Foundation for support (project ABSIDES). We also acknowledge partial support by the Department of Education of the Basque Country Government, the University of the Basque Country (project GV-UPV/EHU, Grant No. IT-366-07), Ministerio de Ciencia y Innovaciòn (Grant No. FIS2010-19609-C02-00). *giorgio.benedek@unimib.it

[1] Y. Guo, Y.-F. Zhang, X.-Yu Bao, T.-Z. Han, Z. Tang, Li-X. Zhang, W.-G. Zhu, E. G. Wang, Q. Niu, Z. Q. Qiu, J.-F. Jia, Z.-X. Zhao, and Qi-K. Xue, Science 306, 1915 (2004).

[2] D. Eom, S. Qin, M. Y. Chou, and C. K. Shih, Phys. Rev. Lett. 96, 027005 (2006).

[3] C. Brun, I-Po Hong, F. Patthey, I. Yu. Sklyadneva, R. Heid, P. M. Echenique, K. P. Bohnen; E. V. Chulkov, and W.-D. Schneider, Phys. Rev. Lett. 102, 207002 (2009).

[4] S. Y. Qin, J. Kim, Q. Niu, and C. K. Shih, Science 324, 1314 (2009).

[5] M. M. Ozer, J. R. Thompson, and H. H. Weitering, Nature Phys. 2, 173 (2006).

[6] T. Zhang, P. Cheng, W.-J. Li, Yu-J. Sun, G. Wang, X.-G. Zhu, K. He, L. Wang, X. Ma, Xi Chen, Y. Wang, Y. Liu, H.Q. Lin, J.-F. Jia, and Qi-K. Xue, Nature Phys. 6, 104 (2010).

[7] B. J. Hinch, C. Koziol, J.P. Toennies, and G. Zhang, Europhys. Lett. 10, 341 (1989).

[8] Y. Jia, B. Wu, H. H. Weitering, and Z. Y. Zhang, Phys. Rev. B 74, 035433 (2006).

[9] R. Otero, A. L. Vazquez de Parga, and R. Miranda, Phys. Rev. B 66, 115401 (2002).

[10] F. Yndurain and M. P. Jigato, Phys. Rev. Lett. 100, 205501 (2008).

[11] J. Braun, P. Ruggerone, G. Zhang, J. P. Toennies, and G. Benedek, Phys. Rev. B 79, 205423 (2009).

[12] M. Jalochowski, M. Hoffmann, and E. Bauer, Phys. Rev. Lett. 76, 4227 (1996).

[13] P. S. Kirchmann, M. Wolf, J.H. Dil, K. Horn, and U. Bovensiepen, Phys. Rev. B 76, 075406 (2007).

[14] J. Kim, S. Qin, W. Yao, Q. Niu, M. Y. Chou, and C-K Shih, Proc. Natl. Acad. Sci. U.S.A. 107, 12761 (2010).

[15] G. Benedek and J. P. Toennies, Surf. Sci. 300, 587 (1994).

[16] R. Arafune et al., Phys. Rev. Lett. 95, 207601 (2005); Phys. Rev. B 80, 073407 (2009).

[17] G. J. Kroes, Science 321, 794 (2008).

[18] H. Gawronski, M. Mehlhorn, and K. Morgenstern, Science 319, 930 (2008).

[19] J. Kliewer, R. Berndt, E. V. Chulkov, V. M. Silkin, P. M. Echenique, and S. Crampin, Science 288, 1399 (2000).

[20] B. Meyer, C. Elsaesser, F. Lechermann, and M. Faehnle, FORTRAN90 Program for Mixed-Basis-Pseudopotential Calculations for Crystals (MPI für Metallforschung, Stuttgart, 1990).

[21] R. Heid and K. P. Bohnen, Phys. Rev. B 60, R3709 (1999).

[22] See Supplemental Material at http://link.aps.org/ supplemental/10.1103/PhysRevLett.107.095502 for details on the derivation of Eqs. (1)-(4) and the band structure of 5 and $7 \mathrm{ML} \mathrm{Pb} / \mathrm{Cu}(111)$ films.

[23] N. Esbjerg and J. K. Norskov, Phys. Rev. Lett. 45, 807 (1980).

[24] G. Grimvall, The Electron-Phonon Interaction in Metals (North-Holland, New York, 1981).

[25] I. Y. Sklyadneva, A. Leonardo, P. M. Echenique, S. V. Eremeev, and E. V. Chulkov, J. Phys. Condens. Matter 18, 7923 (2006).

[26] A. P. Jardine, S. Dworski, P. Fouquet, G. Alexandrowicz, D. J. Riley, G. Y. H. Lee, J. Ellis, and W. Allison, Science 304, 1790 (2004). 